\title{
Max Flesch (1852-1943): Veterinäranatom, Arzt und NS-Opfer
}

\author{
B. Vollmerhaus ${ }^{\star}$, H. Roos, C. Knospe, S. Reese
}

Institut für Tieranatomie, Ludwig-Maximilians-Universität, München

\section{Zusammenfassung}

Der jüdische Arzt Dr. med. Max Flesch, ein Schüler des Würzburger Anatomen Albert von Koelliker (1817-1905), war von 1882-1887 Professor für Anatomie, Histologie und Embryologie an der Tierarzneischule in Bern. Als solcher vereinigte er erstmals an der Schule die drei anatomischen Sparten in einer Hand. Aus seinem Institut ging Oskar Rubeli (1861-1952) hervor, der auch sein Nachfolger wurde. Ab 1888 engagierte sich Max Flesch als praktizierender Arzt und später als Frauenarzt. Im 1. Weltkrieg bewährte er sich als Lazarettarzt. Nach Kriegsende wirkte er vermutlich noch ein Jahrzehnt in seiner Praxis in Frankfurt, bevor er sich auf seinen Altensitz in Hochwaldhausen im hessischen Vogelsberg zurückzog. Flesch publizierte hier seine Erfahrungen als Krankenpfleger bzw. Lazarettarzt aus den Kriegen 1870/71 und 1914-1918. Mit der Machtübernahme durch die Nationalsozialisten änderten sich die Lebensbedingungen für Juden in Deutschland von Grund auf; auch Max Flesch wurde Opfer des Nationalsozialistischen Rassenwahns. Obwohl hoch betagt, wurde er 1942 in das Konzentrationslager Theresienstadt verschleppt und kam dort im Mai 1943 ums Leben. Wir schulden Max Flesch ein ehrendes Gedenken.

Schlüsselwörter: Max Flesch, Veterinäranatomie, Tierarzneischule Bern, Theresienstadt
Max Flesch (1852-1943): Veterinary anatomist, physician and NS-victim

\section{Summary}

The Jewish physician Dr. med. Max Flesch, a student of the Würzburger anatomist Albert von Kölliker (1817-1905), was professor of anatomy, histology and embryology at the School of Veterinary Medicine in Bern from 1882-1887. He was the first at that school who unified the three anatomical fields in one hand. From his Institute came Oskar Rubeli (1861-1952) who was also his successor. From 1888 on Max Flesch was engaged as practitioner and later as gynaecologist. During the First World War he proved his worth as a hospital physician. After the war he most likely was working for another decade in his practice in Frankfurt before retiring in Hochwaldhausen at the Hessian Vogelsberg. During his retirement Flesch published his experiences as 1 nurse and hospital physician, respectively during the wars 1870/71 and 1914-1918. With the assumption of power by the National Socialists the living conditions for Jews in Germany radically changed; also Max Flesch became victim of the Nazi racism. Although very old he was carried off 1942 into the concentration camp Theresienstadt where he lost his life in May 1943. We owe Max Flesch honourable remembrance.

Keywords: Max Flesch, Veterinary Anatomy, Academy of Veterinary Medicine Bern, Theresienstadt 


\section{Persönliche Entwicklung}

Am Neujahrstag des Jahres 1852 wird Max Flesch als Sohn des praktischen Arztes Jacob Gustav Adam Flesch (1819-1892) in Frankfurt am Main geboren. Die ehemalige Reichsstadt nimmt durch die rasche Eingemeindung ihrer Vororte den Charakter einer Großstadt an. Dieser Aufstieg wird durch die günstige Verkehrslage und das Erbe als ehemaliger Geldmarkt begünstigt. Die einsetzende Industrialisierung (Hoechst) zieht Menschenmassen an und sorgt für Verwaltungs-, Verkehrs-, Versorgungs- und Baugestaltungsprobleme. In diesem brodelnden Umfeld erlebt der junge Max Flesch seine Kindheit und Jugend. Wie selbstverständlich wird er durch die wissenschaftlichen und gesellschaftlichen Ambitionen des erfolgreichen Vaters beeinflusst. Dieser ist seit 1841 als praktischer Arzt in Frankfurt am Main niedergelassen und hat sich auf Kinderkrankheiten spezialisiert. Während des Deutschen Kriegs 1866 arbeitet er in einem Militärlazarett, ist Mitbegründer des Ärztlichen Vereins Frankfurt und gründet 1876 dort den ärztlichen Unterstützungsverein. So nimmt es nicht Wunder, dass Max Flesch das Studium der Medizin anstrebt. Er schreibt sich 1869 an der Universität in Bonn ein. Von seinen vorklinischen Lehrern sind zu nennen der intuitive Anatom und Zellforscher Max Schultze (1825-1874), Gründer des «Archiv für mikroskopische Anatomie» und der geniale Physiologe Eduard Pflüger (1829-1910), dessen Name uns noch heute durch sein 1868 gegründetes «Archiv für die gesamte Physiologie» vertraut ist. 1870 bricht der Deutsch-Französische Krieg aus; Max Flesch, der als 18jähriger in eine Fronttruppe nicht aufgenommen wird, meldet sich als freiwilliger Krankenpfleger. Er landet schließlich in einem Johanniter-Korps, hilft im Verwundetendienst und unter anderem auch in der Schlacht von Gravelotte und erhält die Kombattantendenkmünze. Die Erinnerungen an diese Zeit hält er in seiner Monographie (1930, pp. 7-39) kritisch fest, darin Episoden ernster und heiterer Art nicht auslassend. 1871 setzt Max Flesch in Würzburg sein Studium fort. Der bedeutende Pathologe Friedrich Daniel von Recklinghausen und der Histopathologe Köster führen ihn in die pathologische Histologie ein. Sein angefachtes Interesse dokumentiert sich in der ersten wissenschaftlichen Publikation «Über eine besondere Form der Eierstocksgeschwulst», die sich zur Inaugural-Dissertation ausweitet. Zum Sommersemester 1872 wechselt Flesch an die Universität Berlin. Im Herbst 1872, nach Würzburg zurückgekehrt, kann er promovieren und schließt 1873 mit dem Staatsexamen sein Medizinstudium ab.

Flesch leistet seinen Wehrdienst als «einjähriger Arzt» ab, dann bietet ihm 1874 der Anatom von Weltruf Albert von Koelliker (1817-1905) eine Assistentenstelle an seinem Würzburger Institut an. Er nimmt begeistert an und wird bereits 1876 zum Prosektor ernannt, weil der Vorgänger in diesem Amt, Robert Wiedersheim (1848-1923), nach Freiburg wechselte. Flesch publiziert eine Reihe interes- santer Arbeiten und habilitiert sich 1879 für die Fächer Anatomie, Histologie und Entwicklungslehre. Er bleibt noch als Dozent bei Koelliker bis 1882. In diesem Jahr erhält er einen Ruf auf eine Professur für Anatomie, Histologie und Entwicklungslehre an der Tierarzneischule in Bern. Für die damalige Zeit war es nicht ungewöhnlich, dass Humanmediziner, die eine akademische Laufbahn anstrebten, vorübergehend an Tierarzneischulen tätig wurden (Bollinger, Bonnet, Eberth, Rückert u.a.). In allen Fällen war dies für beide Seiten von großem Nutzen gewesen.

\section{Die Professur an der Tierarzneischule Bern (Abb. 1)}

Eine Professur für Anatomie, Histologie und Embryologie ist 1882 in dieser Fächerkombination für Bern neu. Nach dem frühen Tod von Franz Hartmann (1838-1882) war eine Professorenstelle vakant geworden. Hartmann hat neben der Anatomie die Hufbeschlagskunde und das Exterieur vertreten, zeitweise auch die Ambulatorische Klinik geleitet. Die Histologie und Embryologie liegen zusammen mit der Physiologie in den Händen von Balthasar Luchsinger (1849-1886). Dieser gibt mit dem Amtsantritt Flesch's die Histologie und Embryologie an diesen ab. Damit ist jene Fächerkombination gefunden, die sich bis in die Gegenwart bewähren sollte.

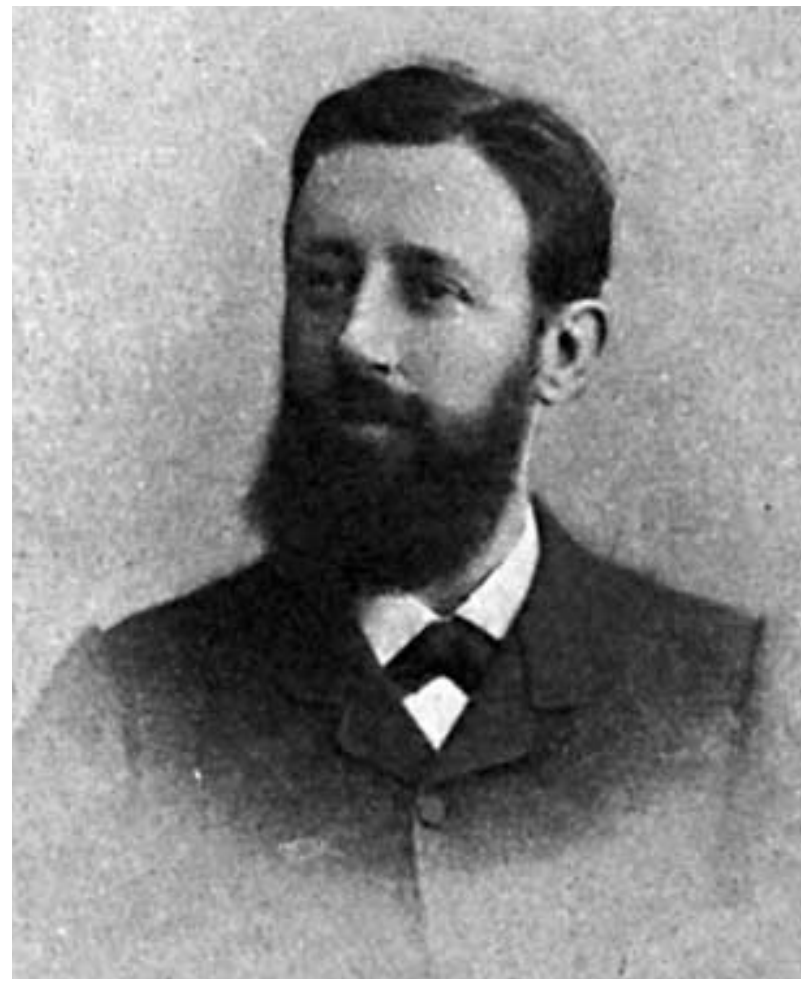

Abbildung 1: Max Flesch um 1885 als Professor für Veterinäranatomie in Bern (aus Fankhauser und Hörning, 1980). 
Max Flesch tritt seine Berner Professur im Herbst 1882 an. Als ein in allen Sparten der Anatomie vorzüglich eingeführter, formvollendeter Redner bekleidet er sein Amt mit großem Geschick (Guillebeau, 1888). Er hält jedoch neben seinem Lehramt an der Tierarzneischule engen Kontakt zur medizinischen Fakultät Bern, indem er dort nebenamtlich als Dozent für Anatomie wirkt. Der dortige Anatom, Prof. Gasser, lädt ihn auch zur Betreuung gemeinsamer Doktoranden ein. Unter diesen ist auch Oskar Rubeli zu finden, ein Assistent Flesch's, der nach seinem Tiermedizinstudium in Bern zusätzlich Medizin studiert. Seine Dissertation trägt den Titel: »Über den Oesophagus des Menschen und verschiedener Haustiere» (Arch. wiss. prakt. Tierheilkd., Berlin 1888). Flesch fördert seinen Schüler in einer Weise, als sei das Habilitationsrecht (das aber erst am 14.05.1900 eingeführt wird) schon der Tierarzneischule verliehen. So wächst in der Person von Rubeli ein potentieller Nachfolger heran.

Flesch führt für sein Institut ein Forschungsmanagement ein, wie er es in Würzburg kennen gelernt hatte. Nicht nur unter seinem eigenen Namen, sondern von zahlreichen Präparanden erscheinen Arbeiten zum Schwerpunktthema Nervensystem, die meist als Dissertationen von der medizinischen Fakultät angenommen werden, Diese Leistungen werden anderenorts aufmerksam registriert, und so kommt es, dass der Dresdner Professor für Physiologie und Histologie an der dortigen Tierarzneischule, Wilhelm Ellenberger, Flesch zur Mitarbeit am «Handbuch der vergleichenden Histologie der Haussäugetiere» auffordert. Dem kommt Flesch mit dem Kapitel «Structur des centralen Nervensystems, des Sympathicus und der peripheren Nerven» im Band I des oben genannten Handbuchs (p.p. 719-756, Parey, Berlin 1887) nach. Max Flesch begründet 1884, zusammen mit Wilhelm Julius Behrens, Göttingen, Leopold Dippel, Darmstadt, und Arthur Wichmann, Utrecht, die «Zeitschrift für wissenschaftliche Mikroskopie und mikroskopische Technik». Er selbst ist einer der fleißigsten Autoren dieses Publikationsorgans. Allein im Band 1 sind fünf, im Band 2 vier eigene Beiträge zur mikroskopischen Technik veröffentlicht. 1892, Jahre nach seinem Wechsel in die Praxis, legt er die Mitherausgeberschaft an besagter Zeitschrift nieder. Die Zeitschrift existierte bis 1970, danach bis heute unter dem Titel «Microscopica acta».

Obwohl Max Flesch nur wenige Jahre der Tierarzneischule Bern angehören sollte, hat er sich doch intensiv mit Fragen der Entwicklung des tierärztlichen Unterrichtswesens auseinandergesetzt. Neben einer neuen Ordnung der Bibliotheksverhältnisse ist ihm besonders an der Verbesserung der Vorbildung der Studierenden und an einer Verleihung von akademischen Graden an ausgebildete Tierärzte gelegen. Dies ist nicht mehr zu seinen Amtszeiten in Bern zu einer Lösung gereift. Aber die Weichen dazu hat er mit manchen Vorschlägen, die auf seiner Erfahrung aus Würzburg gründeten, mit Gleichgesinnten gestellt. Tatsächlich hat wenige Jahre später die Hebung der Vorbildung und Ausbildung der Berner Tier- medizinstudenten dazu geführt, dass die Tierarzneischule als Teil der Universität Bern zu einer Veterinärmedizinischen Fakultät aufsteigt (1. Mai 1900) und alsbald das Promotions- (8. Juni 1900) und Habilitationsrecht (14. Mai 1900) erhält. Für diese Ziele ist Flesch nachweislich (Schweiz. Arch. Tierheilk. 30, 35-43, 1888) eingetreten. Unter Bekundung «seiner Freundschaft und seines anregenden Verkehrs» (Direktor Guillebeau) scheidet Max Flesch mit Jahresende 1887 aus seinem Dienst an der Tierarzneischule in Bern aus. Aber sein Schüler Oskar Rubeli, der die Vorstellungen seines Lehrers aus tiefster Überzeugung teilt und verinnerlicht hat, wirkte, wie wir wissen, in seiner 40-jährigen Dienstzeit mit, diese gesteckten Ziele zu erreichen.

\section{Flesch als Arzt in Frankfurt (Abb. 2)}

Der Grund für den abrupten Abgang Flesch's aus Bern und seinen Entschluss, sich in seiner Vaterstadt Frankfurt/Main als praktizierender Arzt niederzulassen, ist nicht offensichtlich. Dass er enttäuscht ist, in der Wiederbesetzung von Vakanzen auf human-anatomischen Lehrstühlen bisher nicht berücksichtigt worden zu sein, mag angenommen werden. Ob familiäre Unwägbarkeiten eine Rolle spielten, ist nicht belegbar.

Max Flesch wird zum 15. April 1888 als praktischer Arzt in Frankfurt rezipiert. Späterhin wirkt er als Frauenarzt.

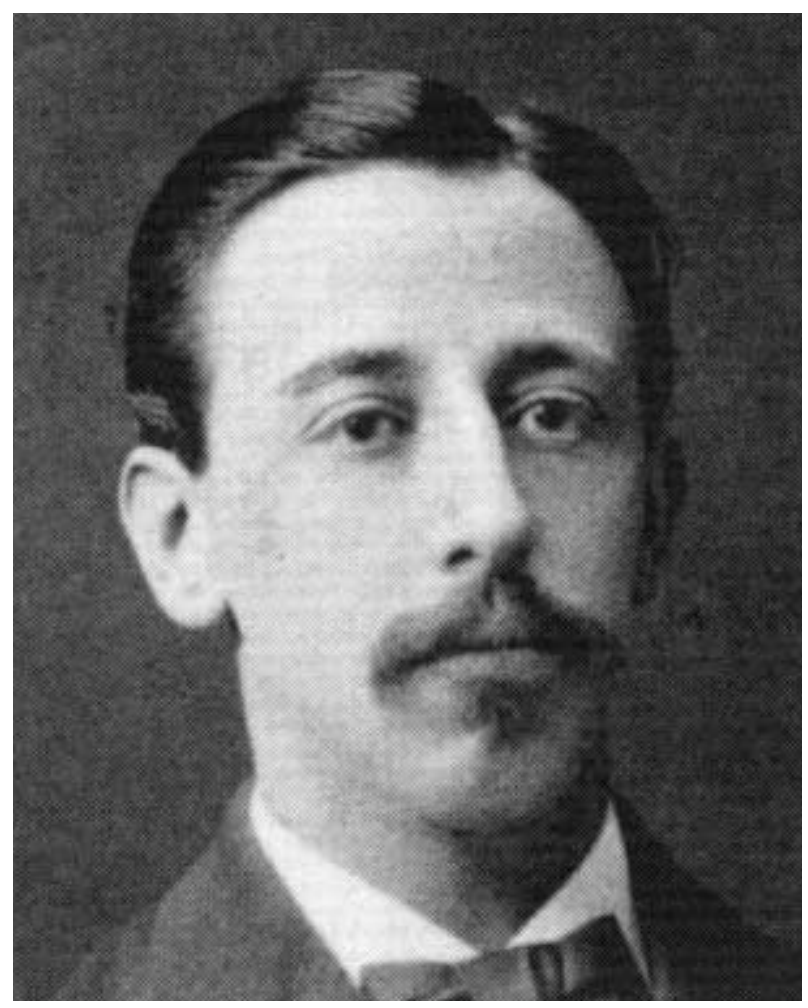

Abbildung 2: Max Flesch Ende des 18. Jahrhunderts als praktizierender Arzt in Frankfurt/ Main (aus Goerttler, 2003). 
Neben seiner praktischen Tätigkeit ist er literarisch tätig und berichtet aus seinen Erfahrungen: 1890 erscheint seine 38-seitige Schrift «Zur Bekämpfung der ansteckenden Krankheiten in den Städten». Eine 78-seitige Schrift (1898 in 2. Aufl.) nimmt sich des Themas «Prostitution und Frauenkrankheiten» an. Schließlich widmet er 1901 eine 43-seitige Schrift dem Problem «Die Hauspflege. Ihre Begründung und Organisation». Max Flesch hat aus der Erfahrung seiner Praxis ein Augenmerk auf die Venerologie, die Lehre von den Geschlechtskrankheiten, gelegt. Zusammen mit dem Juristen Dr. Wertheimer verfasste er 1903 die Schrift «Geschlechtskrankheiten und Rechtsschutz». Die Hinwendung zu diesem Spezialgebiet kommt ihm unter anderem in seiner feldärztlichen Tätigkeit 1914-18 sehr zustatten.

\section{Feldärztliche Tätigkeit 1914-1918}

Mit Ausbruch des 1. Weltkriegs rückt der 62-jährige Max Flesch als freiwilliger Arzt zum Sanitätsdienst ein. Weil er das dienstpflichtige Alter überschritten hat, wird er der Landwehr zugeteilt. Die Zusammenstellung der Kriegslazarettabteilung erfolgt im August 1914 in Würzburg; daraus ergibt sich als Bezeichnung der Truppe die des I. Bayerischen Etappenlazaretts, in dem Flesch im Range eines Oberstabsarztes (Abb. 3) die Aufgaben eines Chefarztes übertragen werden. 1916 reicht Flesch ein Abschiedsgesuch wegen seines vorgerückten Alters ein, das aber abschlägig beschieden wird. Kurz vor Kriegsende wird seine Einheit der II. Preußischen Armee zugeordnet, aber da setzen die Auflösungserscheinungen mit Blick auf das Kriegsende bereits ein. Im November 1918 wird Flesch zur Abwicklung seiner Einheit von St. Nicklas nach München, dem Standort des I. Bayerischen Etappenlazaretts, das nun wieder maßgeblich ist, in Marsch gesetzt. Über Würzburg erfolgt seine Entlassung aus der Landwehr, und er muss sich nach $41 \frac{1}{2}$ Jahren feldärztlicher Tätigkeit mit 67 Jahren der schweren Aufgabe unterziehen, seine verlorene Praxis in Frankfurt/M. wieder aufzubauen.

\section{Häftling im Konzentrationslager Theresienstadt 1942/43}

Das Ehepaar Flesch hat sich zwischen 1924 und 1927 in die Stille des hessischen Vogelbergs zurückgezogen, in der Hoffnung einen unbeschwerten, sorgenfreien Lebensabend $\mathrm{zu}$ verbringen. Max Flesch ist wieder schriftstellerisch rege tätig (Abb. 4). Aber die Welt hat sich verändert. In Deutschland herrscht der Nationalsozialismus und die neuen Machthaber haben rassenpolitische und gesetzgeberische Maßnahmen in Kraft gesetzt, die zuerst die Vertreibung der Juden aus Deutschland und später deren Abtransport in die Vernichtungslager zum Ziel haben. Die Nürnberger Rassengesetze (15.9.1935) und die Pläne der Wannseekonferenz (20.1.1942) sind Eckdaten, die den

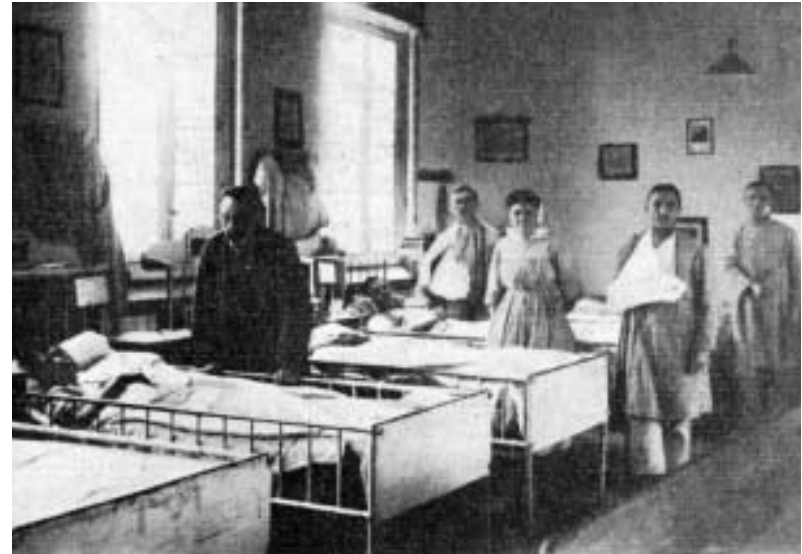

Abbildung 3: Max Flesch als Oberstabsarzt bei einer Visite im Lazarett Tourcoing (aus Flesch, 1930).

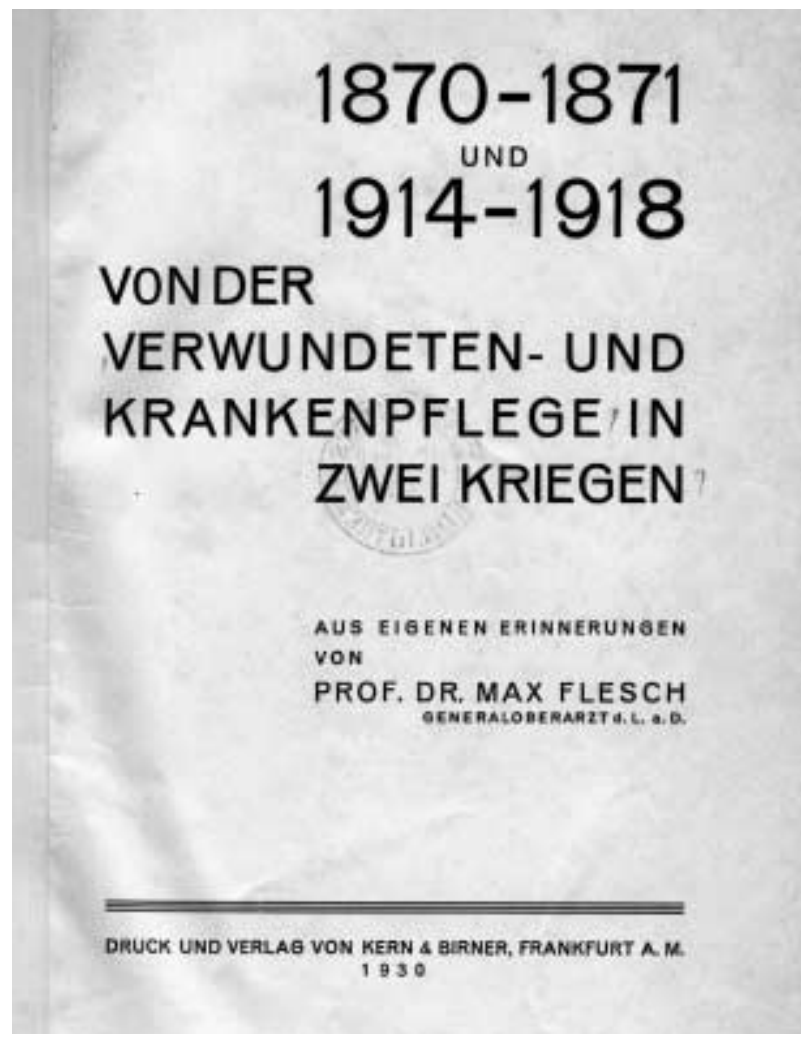

Abbildung 4: Titelblatt seines Erinnerungsbuchs 1930.

Rassenwahn und das Vernichtungswerk der Nazis dokumentieren. Unter den Konzentrationslagern für politisch und rassisch Verfolgte hat Theresienstadt die Funktion eines Durchgangs- und Konzentrationslagers für Juden, zunächst aus der Region des Protektorats Böhmen und Mähren (errichtet nach dem Münchner Diktat vom 14./15.3.1939), später für Juden aus Deutschland (sog. Altreich) und Österreich (sog. Ostmark) sowie im Zuge des fortschreitenden Krieges aus allen Teilen Europas. Welches individuelle Schicksal Max Flesch als Häftling 
in diesem Lager erleiden musste, ist nicht bekannt. Aber die allgemein bekannt gewordenen Zustände in Theresienstadt lassen erahnen, welchen Zwangseinflüssen Max Flesch, inzwischen 90-jährig, ausgesetzt war.

Theresienstadt (tschechisch: Terezin) wurde Ende des 18. Jahrhunderts von Kaiser Josef II von Österreich als befestigte Garnisonsstadt gegründet und nach dessen Mutter Kaiserin Maria Theresia benannt. Die Nationalsozialisten errichteten hier im 2. Weltkrieg ein Konzentrationslager, das sie draußen in der Welt als «jüdische Mustersiedlung» deklarierten, um den Verbleib zum Teil prominenter Juden als «Wohnsitzverlagerung» zu verschleiern. Deshalb wurde das Internierungslager, das sich innerhalb der gut $\mathrm{zu}$ überwachenden Befestigungsanlagen befand, verharmlosend als «Ghetto» bezeichnet. Die Errichtung des Lagers erfolgte im November 1941 durch 342 jüdische Handwerker aus der Region. Mitte Dezember hatte sich die Zahl der Häftlinge auf 7'350 erhöht, die aus der Tschechoslowakei stammten. Im April 1942 waren es 15'732 Zwangs eingewiesene Juden. Ab Juni 1942 setzten die Transporte aus Deutschland ein. Sie wurden in verschiedenen Großstädten zusammengestellt. Von Frankfurt/M. aus gingen 1942 drei Großtransporte ab: 18. August, 1. und 15. September. Die ersten beiden Transporte bildeten Juden aus Frankfurt selbst. Im dritten Transport (15.9.42) wurden Juden auch aus der Umgebung verschleppt, so dass wir annehmen, dass dies das Verschleppungsdatum des Ehepaars Flesch ist. In den Jahren 1943 und 1944 wurden noch 7 kleinere Gruppen von Frankfurt aus nach Theresienstadt deportiert. Schon der jeweils eintägige Transport in Bahnwaggons forderte erste Todesopfer. Die Neueingewiesenen wurden nach Geschlechtern getrennt untergebracht. Sie hatten ihren «Aufenthalt» mit sog. «Heimeinkaufsverträgen» selbst zu finanzieren, um sie ihres Vermögens zu berauben. Das Lager stand unter dem Kommando der Geheimen Staatspolizei (Gestapo), die einen «Ältestenrat» aus Juden ernannte, der das Leben im Lager organisieren sollte. Das Wachpersonal setzte sich aus tschechischen Gendarmen zusammen. Hunger, Krankheit, Entkräftung und Tod beherrschten das Lagerleben. Das Eingepferchtssein auf engstem Raum durch die rasante Zunahme der Inhaftierten nahm ihnen die Möglichkeit, gesund zu bleiben und erhöhte die Gefahren der Erkrankung. Der «Daseinsraum» im Lager wurde systematisch durch einen «Sterberaum» ersetzt. Insgesamt haben 140'000 Menschen das Lager durchlaufen; von diesen stammten 42'000 aus Deutschland. Im Laufe der Jahre zwischen 1941 bis 1945 wurden 86'934 Häftlinge in Arbeits- oder Vernichtungslager weiter deportiert. Unter denjenigen, die laut behördlicher Bestimmungen im Lager zu bleiben hatten, befanden sich Überalterte und Personen mit besonderen Verdiensten, vornehmlich aus dem 1. Weltkrieg. Insgesamt starben 33'521 der Inhaftierten an Erschöpfung und Krankheiten. Unter ihnen ist Max Flesch zu verzeichnen, der am 6. Mai 1943, 91-jährig, ums Leben kam. Über Flesch's Ehefrau, die gleichfalls nach Theresienstadt verschleppt wurde (Goerttler, 2006), ist nichts bekannt. Zum Verbleib des gemeinsamen Sohnes Hans (geb. 18.12.1896), der am 1. Weltkrieg teilgenommen hat (Flesch, 1930, p. 88, Hinweis auf eine Erkrankung im Feld), sind laut einer Erinnerungstafel des Jüdischen Museums Frankfurt/M. Deportationsort und Sterbedatum unbekannt. Ein weiteres gemeinsames Kind der Eheleute Flesch ist Ende des 1. Weltkriegs gestorben (Flesch, 1930, p. 227). Von den Häftlingen in Theresienstadt erlebten 19'161 am 8. Mai 1945 die Befreiung.

Max Flesch gehört wegen seiner relativ kurzen Wirkungszeit an der Berner Tierarzneischule zu den (fast) vergessenen Persönlichkeiten unseres Faches. Bei Betrachtung seines faszinierenden Schaffens dürfen wir ihn zu den Erfolgreichen, Fortschrittlichen und Zukunftweisenden in der Veterinärmedizin zählen. Sein tragisches Lebensende verpflichtet uns, die Erinnerung an sein verdienstvolles Wirken und sein finales Leiden immerdar wach zu halten. 


\section{Literatur}

Adler, H. G.: Theresienstadt 1941-45. Das Antlitz einer Zwangsgemeinschaft. Verlag J. C. B. Mohr (Paul Siebeck), Tübingen, 1955.

Anonym: Jüdisches Museum Frankfurt/M.: «und keiner hat für uns kaddisch gesagt...», Deportationen aus Frankfurt am Main 1941 bis 1945. Stroemfeld-Verlag, Frankfurt, 2004.

Fankhauser, R. und B. Hörning: 175 Jahre tierärztliche Lehranstalt zu Bern. Schweiz. Arch. Tierheilk. 1980, 122: 57-94.

Goerttler, K.: Wegbereiter unserer naturwissenschaftlich-medizinischen Moderne. 219 Biographien zur Portrait-Sammlung des Anatomen Rudolf Wiedersheim. Verlag Academic-Press/ Studenten-Presse GmbH, Heidelberg, 2003.

Goerttler, K.: Briefliche Mitteilung zur Chronik Flesch vom 20.03.2006, Heidelberg, 2006.

Guillebeau, A.: Rücktritt von Prof. Flesch - Ernennung von Herrn O. Rubeli. Schweiz. Arch. Tierheilk. 1888, 30: 100-101. Kallmorgen, W.: Siebenhundert Jahre Heilkunde in Frankfurt am Main. Verlag Moritz Diesterweg, Frankfurt/M., 1936.

Kramer, R.: Nachschaffende Musiker. In: Juden im Deutschen Kulturbereich, ein Sammelwerk. Hrsg. S. Kaznelson, Jüdischer Verlag, 2. Aufl., Berlin, 1959.

Ottenheimer, H.: Soziale Arbeit. In: Juden im Deutschen Kulturbereich, ein Sammelwerk. Hrsg. S. Kaznelson, Jüdischer Verlag, 2. Aufl., Berlin, 1959.
Rubeli, O.: Die Tierärztliche Lehranstalt zu Bern in den ersten hundert Jahren ihres Bestehens. Festschrift, Haller'sche Buchdruckerei, Bern, 1906.

Vollmerhaus, B., H. Roos, S. Reese und C. Knospe: Kleine Chronik der Veterinäranatomie im deutschen Sprachraum, mit einer Sammlung von Kurzbiographien, katalogisiert nach Bildungsstätten. Eigenverlag, Druck: Hausdruckerei der Ludwig-Maximilians-Universität, München, 2007.

Eine Liste der Veröffentlichungen von Max Flesch kann an der Korrespondenzadresse angefordert werden.

\section{Korrespondenzadresse}

Prof. Dr. Bernd Vollmerhaus

Institut für Tieranatomie I

Veterinärstr. 13

D80539 München

Tel: 00498921802568

Fax 00488921803202

Email: sven.reese@lmu.de

Manuskripteingang: 24. August 2007

Angenommen: 10. Oktober 2007 